\title{
Single-port Laparoscopy with Modified Needle Hook-assisted Extracorporeal High Ligation of Hernia Defects (Suwardi's Technique): An Experience from Single-center Hospital
}

\author{
Alfian Fahmy (iD, Galih Santoso Putra (i), Muhammad David Perdana Putra (D), Suwardi Suwardi*(D) \\ Department of Surgery, Sebelas Maret University, Surakarta, Central Java, Indonesia
}

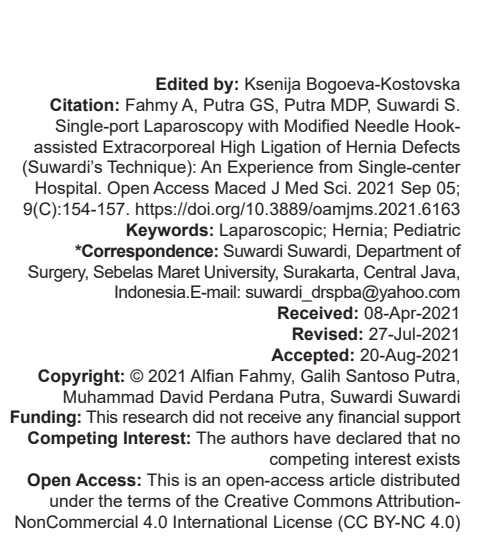

Introduction

In 1997, El Gohary first reported laparoscopic action on hernia repair in a female patient. Since then, laparoscopic inguinal hernia ligation has been performed in various medical institutions with a variety of methods including closing the defect intraperitoneally and extracorporeal ligation of the herniated ring defect [1].

At present, it has been described that laparoscopic hernia repair with extracorporeal ligation as compared to intraperitoneal suturing results in a significant reduction in intraoperative time, a lower recurrence rate, and excellent cosmetic results [2]. This article discusses the technique of extracorporeal ligation of an inguinal hernia assisted by two epidural needles that are formed into hooks used percutaneously so as to minimize injury to the skin, muscles, and nerves.

\section{Case Presentation}

Data were taken from January 2016 to December 2020 in the Pediatric Surgery Department of Dr. Moewardi General Hospital, Surakarta. There were 22 cases, consisting of 19 boys and 3 girls aged 1-14 years (mean 5 years) underwent singleport laparoscopy with modified extracorporeal ligation of hernia defects using epidural needles formed into hooks (Suwardi's technique).

\section{Suwardi's technique}

Insert the infraumbilical Veress needle, then insert the infraumbilical $5 \mathrm{~mm}$ trocar as a laparoscope camera (Figure 1). Identify herniated defects in the right and left inguinal rings. The landmark for hook needle insertion is midway between the umbilicus and spina iliaca anterior superior on the ipsilateral side of the hernia defect where the needle hook is inserted (Figure 2). Laparoscopic light-guided, 17 G epidural needle (Terumo, diameter $1.40 \mathrm{~mm}$, length $80 \mathrm{~mm}$ ) is inserted and traversed along the bottom half of the internal inguinal ring (Figure 3). The needle is then traversed extraperitoneally under the testicular blood vessel, the spermatic duct, without performing a hydrodissection between the pre-peritoneal spaces and removing the peritoneum (Figure 4).

In this technique, hydrodissection is not performed, which usually involves infusion of normal 
saline into the pre-peritoneal tissue because it reduces visualization of structures in the peritoneum (vasa and vas deferens). The non-absorbable 2.0 monofilament thread is passed through the lumen of the epidural needle inside the abdominal cavity and the outside the skin.

The non-absorbable thread is then pulled in a loop through the lumen of the epidural needle. The epidural needle is then removed from the abdomen along with the thread. The defect in the hernial ring is then closed and the loop in the peritoneum is tied extracorporeally. This approach places the knot over the fascia. This procedure is done in succession through one needle prick. If there is a defect on the contralateral, the same action on the contralateral side is performed in the same procedure.

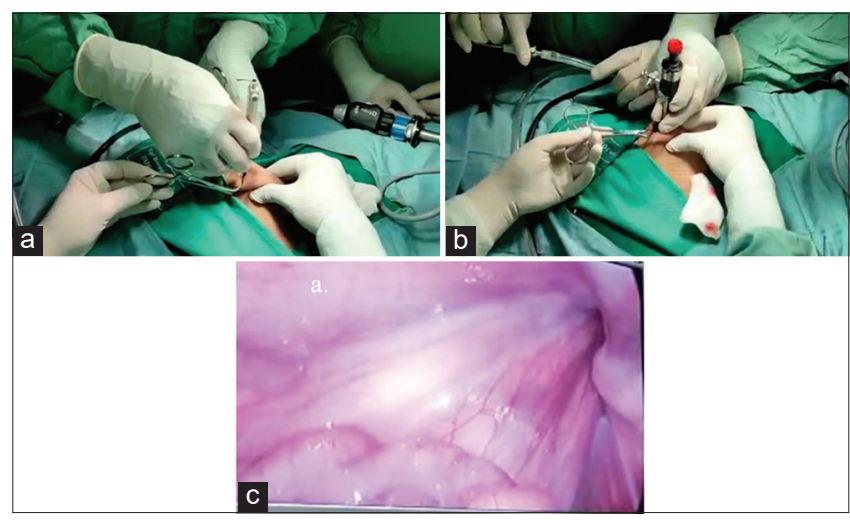

Figure 1: (a) Insertion of the Veress needle into the infraumbilical. (b) Laparoscope port insertion. (c) Evaluate the inguinal canal, in this case, the defect is in the left inguinal canal

\section{Results}

The clinical characteristics of the patients are shown in Table 1. The mean follow-up period was 6 months. The 22 patients were treated with a single port without the aid of forceps. This single-port laparoscopic technique with extracorporeal modification with an epidural needle can evaluate the contralateral side, close the hernia defect, and shorten the duration of surgery. All patients were carried out on an outpatient basis within $24 \mathrm{~h}$ postoperatively without any postoperative complications.

\section{Discussion}

In 1995, Takehara et al. developed an extraperitoneal laparoscopy/laparoscopic percutaneous extraperitoneal closure (LPEC) to treat inguinal hernias in children. This technique is performed by placing the laparoscope through the infraumbilical incision, then grasping the forceps through the trocar on the right or left infraumbilical side. Then, a LPEC needle with a thread is inserted percutaneously in the inguinal line into the abdominal cavity. The hernia bag that is still

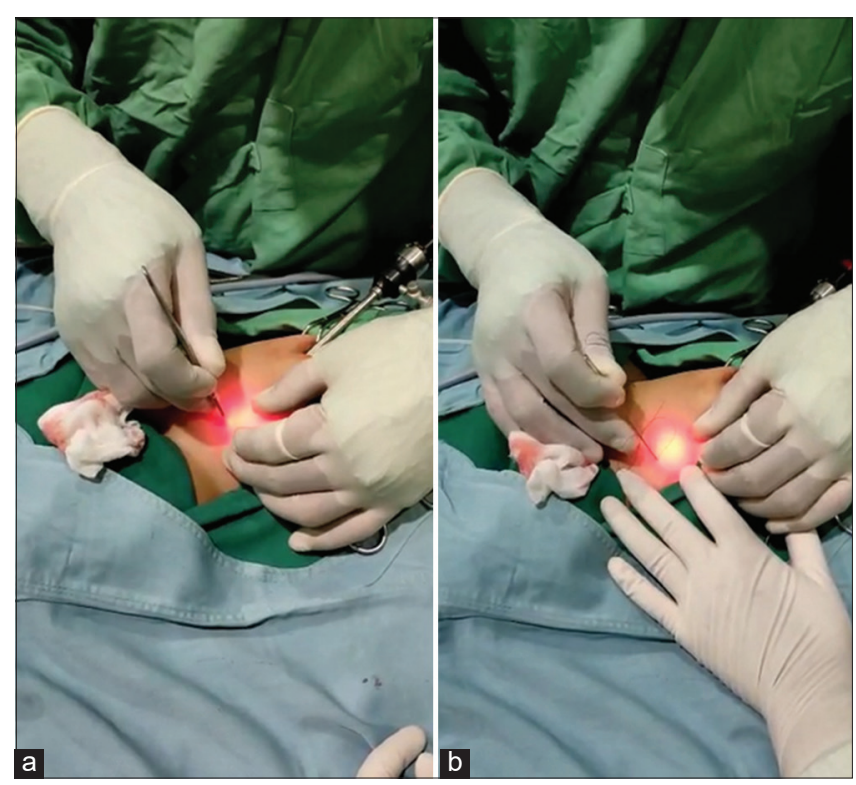

Figure 2: (a) Incision on the inguinal skin using mess no. 11 (b) Insertion of the epidural needle through the previous incision wound

Table 1. Clinical characteristics of patients

\begin{tabular}{|c|c|c|c|c|c|c|c|}
\hline Patient & Sex & Age (years) & Side of the hernia & Contralateral hernia & Pneumoperitoneum timing & Complications & Follow-up \\
\hline 1 & $\mathrm{~L}$ & 1 & Right & None & 34 & - & 6 \\
\hline 2 & L & 1 & Right & Present & 31 & - & 6 \\
\hline 3 & L & 1 & Right & None & 40 & - & 6 \\
\hline 4 & L & 3 & Right & None & 29 & - & 6 \\
\hline 5 & P & 3 & Right & None & 25 & - & 6 \\
\hline 6 & L & 3 & Left & None & 32 & - & 6 \\
\hline 7 & L & 3 & Right & None & 25 & - & 6 \\
\hline 8 & L & 3 & Right & None & 30 & - & 6 \\
\hline 9 & $\mathrm{P}$ & 3 & Left & None & 29 & - & 6 \\
\hline 10 & L & 4 & Left & None & 31 & - & 6 \\
\hline 11 & L & 4 & Right & Present & 27 & - & 6 \\
\hline 12 & L & 4 & Right & None & 45 & - & 6 \\
\hline 13 & P & 5 & Left & None & 47 & - & 6 \\
\hline 14 & L & 5 & Right & None & 41 & - & 6 \\
\hline 15 & L & 6 & Right & None & 28 & - & 6 \\
\hline 16 & L & 7 & Right & None & 29 & - & 6 \\
\hline 17 & L & 7 & Right & Present & 31 & - & 6 \\
\hline 18 & L & 7 & Left & None & 38 & - & 6 \\
\hline 19 & L & 8 & Right & None & 38 & - & 6 \\
\hline 20 & L & 8 & Right & Present & 35 & - & 6 \\
\hline 21 & L & 9 & Right & None & 34 & - & 6 \\
\hline 22 & L & 14 & Right & None & 32 & - & 6 \\
\hline
\end{tabular}




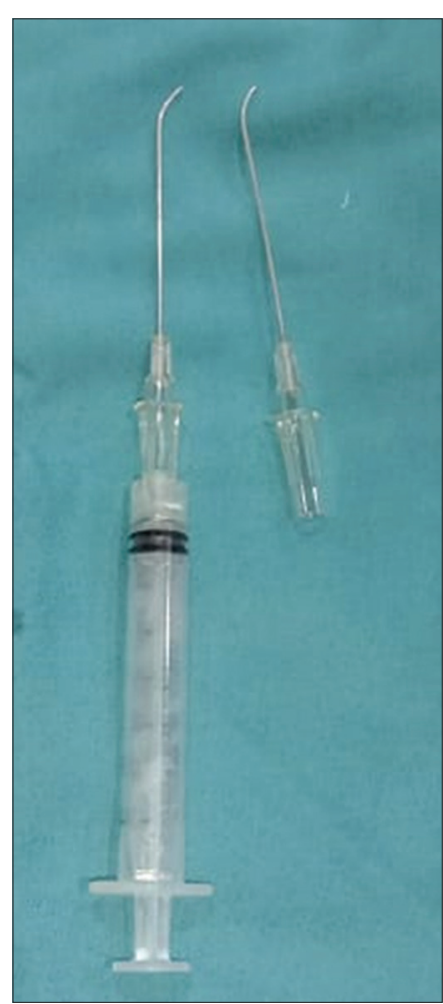

Figure 3: Modified epidural needle

open is closed extraperitoneally with a suture that is looped around the internal inguinal ring using an LPEC needle. This approach allows the surgeon to carry out contralateral exploration without adding an additional port followed by closure of the processus vaginalis [3].

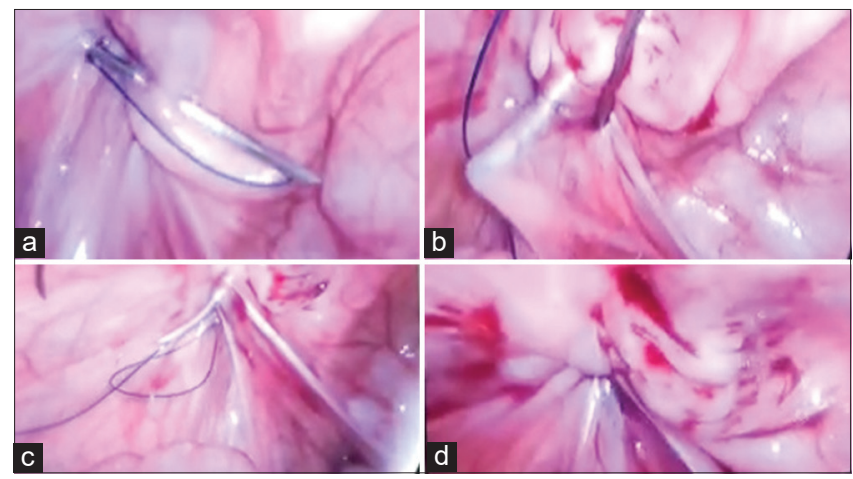

Figure 4: (a) The epidural needle is passed through the peritoneum right at the inguinal defect. (b) The second epidural needle passes through the peritoneum, circling the defect from the inguinal. (c) Loop through the second needle. (d) Closed defect

Tatekawa described a laparoscopic technique assisted by one grasping forceps to close the hernia ring using a non-absorbable monofilament thread that is inserted into the abdominal cavity through a needle on one side of the inguinal ring and pulled out through the other side with a needle shaped like a hook. The knot is made outside the abdominal cavity [2].

As another modification, this technique is assisted by injection of normal saline into the preperitoneal space to prevent injury to the blood vessels in the peritoneum [2]. In extracorporeal modified single-port laparoscopy using a hook-shaped epidural needle without the aid of a forceps, it is important to prevent nerve and muscle injury that can occur during circular suturing of the herniated ring. This technique does not use hydrosection because it interferes with the visualization of blood vessels in the peritoneal wall. It is also important that the peritoneum is completely closed at the bottom of the ring so that it does not leave a gap between the vas deferens and the ligaments. If there is a gap, it can be closed approximately. Since the tool used to insert the thread is a modified epidural needle in the shape of a hook without completely removing the needle blade, there is little chance of nerve and muscle injury over the circular suture area. In addition to, damage to the spermatic chorda can be prevented by hydrodissection in the pre-peritoneal space. However, hydrodissection is not performed in this procedure because it affects the identification of blood vessels on the peritoneal surface [4], [5], [6], [7].

\section{Conclusion}

This case report describes a single-port extracorporeal ligation technique assisted by an epidural needle shaped like a hook to close the inguinal hernia defect (Suwardi's technique). This technique is cheap enough to perform with minimal complications. This technique provides a shorter operating time and satisfying cosmetic results.

\section{References}

1. El-Gohary MA. Laparoscopic Ligation of Inguinal Hernia in Girls. Pediatr Endosurgery Innov Tech. 1997;1(3):185-8. https://doi. org/10.1089/pei.1997.1.185

2. Tatekawa Y. Laparoscopic extracorporeal ligation of hernia defects using an epidural needle and preperitoneal hydrodissection. J Endourol. 2012;26(5):474-7. https://doi. org/10.1089/end.2011.0498

PMid:22168769

3. Takehara H, Yakabe S, Kameoka K. Laparoscopic percutaneous extraperitoneal closure for inguinal hernia in children: Clinical outcome of 972 repairs done in 3 pediatric surgical institutions. J Pediatr Surg. 2006;41(12):1999-2003. https://doi. org/10.1016/j.jpedsurg.2006.08.032

PMid: 17161191

4. Chang YT. Technical refinements in single-port laparoscopic surgery of inguinal hernia in infants and children. Diagn Ther Endosc. 2010;2010:392847. https://doi. org/10.1155/2010/392847 PMid:20508740

5. Chang YT, Wang JY, Lee JY, Chiou CS. A simple single-port laparoscopic-assisted technique for completely enclosing inguinal hernia in children. Am J Surg. 2009;198(1):e13-6. https://doi.org/10.1016/j.amjsurg.2008.11.047 
PMid:19555784

6. Chang YT, Wang JY, Lee JY, Chiou CS, Hsieh JS. Onetrocar laparoscopic transperitoneal closure of inguinal hernia in children. World J Surg. 2008;32(11):2459-63. https://doi. org/10.1007/s00268-008-9732-4

PMid:18791861
7. Shalaby R, Ismail M, Dorgham A, Hefny K, Alsaied G, Gabr K, et al. Laparoscopic hernia repair in infancy and childhood: Evaluation of 2 different techniques. J Pediatr Surg. 2010;45(11):2210-6. https://doi.org/10.1016/j. jpedsurg.2010.07.004

PMid:21034946 